\title{
TWISTED CONJUGACY IN BRAID GROUPS
}

\author{
JUAN GONZÁLEZ-MENESES AND ENRIC VENTURA
}

\begin{abstract}
In this note we solve the twisted conjugacy problem for braid groups, i.e. we propose an algorithm which, given two braids $u, v \in B_{n}$ and an automorphism $\varphi \in \operatorname{Aut}\left(B_{n}\right)$, decides whether $v=(\varphi(x))^{-1} u x$ for some $x \in B_{n}$. As a corollary, we deduce that each group of the form $B_{n} \rtimes H$, a semidirect product of the braid group $B_{n}$ by a torsion-free hyperbolic group $H$, has solvable conjugacy problem.
\end{abstract}

\section{INTRODUCTION}

Let $G$ be a group, and $\varphi \in A u t(G)$ an automorphism (which we shall write on the left of the argument, $g \mapsto \varphi(g))$. We say that two elements $u, v \in G$ are $\varphi$-twisted conjugated, denoted $u \sim_{\varphi} v$, if there exists $x \in G$ such that $v=(\varphi(x))^{-1} u x$. It is straightforward to see that $\sim_{\varphi}$ is an equivalence relation on $G$, which coincides with standard conjugation in the case $\varphi=I d$ (we shall use the symbol $\sim$ instead of $\sim_{I d}$ ). Reidemeister was the first author considering this concept (see [14]), which has an important role in modern Nielsen fixed point theory.

As one might expect, in general, twisted conjugacy classes are much more complicated to understand than standard conjugacy classes in a group $G$. For instance, algorithmic recognition of them already presents big differences. The twisted conjugacy problem for a group $G$ consists on finding an algorithm which, given an automorphism $\varphi \in \operatorname{Aut}(G)$ and two elements $u, v \in G$, decides whether $u \sim_{\varphi} v$ or not. While the conjugacy problem (i.e. the $I d$-twisted conjugacy problem) is very easy for free groups, both conceptually and computationally, the twisted conjugacy problem is solvable but much harder in both senses, see Theorem 1.5 in [3].

Of course, a positive solution to the twisted conjugacy problem automatically gives a solution to the (standard) conjugacy problem, which in turn provides a solution to the word problem. The existence of a finitely presented group $G$ with solvable word problem but unsolvable conjugacy problem is well known (see [13]).

2000 Mathematics Subject Classification. 20F36, 20F10.

Key words and phrases. Braid group, twisted conjugacy. 
In the same direction, there exists a finitely presented group with solvable conjugacy problem, but unsolvable twisted conjugacy problem (see Corollary 4.9 in $[2])$.

A subgroup $A \leqslant A u t(G)$ is said to be orbit decidable if there is an algorithm which, given two elements $u, v \in G$ as input, decides whether one can be mapped to the other up to conjugacy, by some automorphism in $A$, i.e. whether $v \sim \alpha(u)$ for some $\alpha \in A$ (see [2] for more details). For example, the conjugacy problem in $G$ coincides precisely with the orbit decidability of the trivial subgroup $\{I d\} \leqslant$ $\operatorname{Aut}(G)$.

Let

$$
1 \longrightarrow F \stackrel{\alpha}{\longrightarrow} G \stackrel{\beta}{\longrightarrow} H \longrightarrow 1 .
$$

be a short exact sequence of groups. Since $\alpha(F)$ is normal in $G$, for every $g \in G$, the right conjugation $\gamma_{g}$ of $G$ induces an automorphism of $F, x \mapsto g^{-1} x g$, which will be denoted $\varphi_{g} \in A u t(F)$ (note that, in general, $\varphi_{g}$ does not belong to $\operatorname{Inn}(F))$. It is clear that the set of all such automorphisms,

$$
A_{G}=\left\{\varphi_{g} \mid g \in G\right\}
$$

forms a subgroup of $\operatorname{Aut}(F)$ containing $\operatorname{Inn}(F)$. We shall refer to it as the action subgroup of the given short exact sequence.

Such a sequence is said to be algorithmic provided it is given along with algorithms: (i) to compute in the groups $F, G$ and $H$ (i.e. multiply and invert elements), and compute images under $\alpha$ and $\beta$; (ii) to compute one pre-image in $G$ of any given element in $H$; and (iii) to compute pre-images in $F$ of elements in $G$ mapping to the trivial element in $H$. The typical example (though not the unique one) of an algorithmic short exact sequence occurs when groups are given by finite presentations and maps are given by images of generators. In fact, (i) is immediate, we can use the positive part of the membership problem for $\beta(G)$ in $H$ to compute pre-images in $G$ of elements in $H$, and use the positive part of the membership problem for $\alpha(F)$ in $G$ to compute pre-images in $F$ of elements in $G$ mapping to $1_{H}$ (see Section 2 in [2]).

Assuming certain conditions on the groups $F$ and $H$, the main result in [2] establishes the following characterization of the solvability of the conjugacy problem for $G$, in terms of the orbit decidability for the corresponding action subgroup.

Theorem 1.1 (Bogopolski, Martino, Ventura [2]). Let

$$
1 \longrightarrow F \stackrel{\alpha}{\longrightarrow} G \stackrel{\beta}{\longrightarrow} H \longrightarrow 1
$$

be an algorithmic short exact sequence of groups such that

(i) F has solvable twisted conjugacy problem,

(ii) $H$ has solvable conjugacy problem, and 
(iii) for every $1 \neq h \in H$, the subgroup $\langle h\rangle$ has finite index in its centralizer $C_{H}(h)$, and there is an algorithm which computes a finite set of coset representatives, $z_{h, 1}, \ldots, z_{h, t_{h}} \in H$,

$$
C_{H}(h)=\langle h\rangle z_{h, 1} \sqcup \cdots \sqcup\langle h\rangle z_{h, t_{h}} .
$$

Then, the conjugacy problem for $G$ is solvable if and only if the action subgroup $A_{G} \leqslant \operatorname{Aut}(F)$ is orbit decidable.

Many groups satisfy conditions (ii) and (iii); for example, they are easily verified for a finitely generated free group, and with a bit more work, they can also be proven for torsion-free hyperbolic groups, see Proposition 4.11 in [2].

On the other hand, solvability of the twisted conjugacy problem is a stronger condition on $F$. In this sense, the introduction of [2] contains the following comment: "In light of Theorem 1.1, it becomes interesting, first, to collect groups $F$ where the twisted conjugacy problem can be solved. And then, for every such group $F$, to study the property of orbit decidability for subgroups of Aut $(F)$ : every orbit decidable (undecidable) subgroup of Aut $(F)$ will correspond to extensions of $F$ having solvable (unsolvable) conjugacy problem".

The goal of the present paper is to contribute a new result into this direction, taking as a base group the braid group, $F=B_{n}$.

Consider the braid group on $n$ strands, given by the classical presentation

$$
B_{n}=\left\langle\begin{array}{l|ll}
\sigma_{1}, \sigma_{2}, \ldots, \sigma_{n-1} & \begin{array}{ll}
\sigma_{i} \sigma_{j}=\sigma_{j} \sigma_{i} & |i-j|>1 \\
\sigma_{i} \sigma_{j} \sigma_{i}=\sigma_{j} \sigma_{i} \sigma_{j} & |i-j|=1
\end{array}
\end{array}\right\rangle
$$

It is well known that the conjugacy problem is solvable in $B_{n}$. The first, nonefficient solution was given by Garside [8]. It was subsequently improved in $[6,7$, $1,9,10]$, in such a way that the current solution is very efficient in most cases.

Theorem 1.2 (Garside [8]). The conjugacy problem is solvable in $B_{n}$.

Also, the automorphism group of $B_{n}$ is quite well understood. Among other results, the following one will be crucial for our argumentation.

Theorem 1.3 (Dyer, Grossman [5]). Let $B_{n}$ be the braid group on $n$ strands. Then $\left|\operatorname{Out}\left(B_{n}\right)\right|=2$. More precisely, Aut $\left(B_{n}\right)=\operatorname{Inn}\left(B_{n}\right) \sqcup \operatorname{Inn}\left(B_{n}\right) \cdot \varepsilon$, where $\varepsilon: B_{n} \rightarrow B_{n}$ is the automorphism which inverts each generator, $\sigma_{i} \mapsto \sigma_{i}^{-1}$.

Using the above two results, we will solve the twisted conjugacy problem in $B_{n}$, and the orbit decidability problem for every subgroup $A \leqslant A u t\left(B_{n}\right)$. As a consequence, we deduce that the conjugacy problem is solvable in certain extensions of $B_{n}$. 
Theorem 4.9. The twisted conjugacy problem is solvable in the braid group $B_{n}$.

Theorem 5.1. Every finitely generated subgroup $A \leqslant A u t\left(B_{n}\right)$ is orbit decidable.

Theorem 5.2. Let $G=B_{n} \rtimes H$ be an extension of the braid group $B_{n}$ by a finitely generated group $H$ satisfying conditions (ii) and (iii) above (for instance, take $H$ torsion-free hyperbolic). Then, $G$ has solvable conjugacy problem.

The structure of the paper is as follows. In Section 2 we review some known facts about normal forms for braids that will be used later. In Section 3 we determine a well defined finite subset of each $\varepsilon$-twisted conjugacy class in $B_{n}$. And in Section 4 we give an algorithm to construct such set from a given element in the class, solving the twisted conjugacy problem in $B_{n}$. Finally, in Section 5 we solve the orbit decidability problem for subgroups of $A u t\left(B_{n}\right)$ and conclude Theorem 5.2.

\section{NORMAL FORMS OF BRAIDS}

In this section we will recall the notion of normal form for braids, as explained in [15, Chapter 9] and [6], and we shall also provide some technical lemmas that will be used to prove our main results.

In the braid group $B_{n}$, an element is called positive if it can be written as a product of non-negative powers of the generators $\sigma_{1}, \ldots, \sigma_{n-1}$. It turns out that if we regard the standard presentation of the braid group (1) as a monoid presentation, it yields a monoid $B_{n}^{+}$which embeds in $B_{n}$, and is precisely the submonoid of positive braids [8]. This means that two positive words represent the same braid if and only if one can be obtained from the other by a finite sequence of the following operations: Either replacing a subword $\sigma_{i} \sigma_{j}$ by $\sigma_{j} \sigma_{i}$ for $|i-j|>1$, or replacing a subword $\sigma_{i} \sigma_{j} \sigma_{i}$ by $\sigma_{j} \sigma_{i} \sigma_{j}$ for $|i-j|=1$.

There is a partial order $\preccurlyeq$ on the elements of $B_{n}$, called the prefix order, defined by $a \preccurlyeq b$ if and only if $a^{-1} b$ is positive. If $a$ and $b$ are positive this means that $b$ can be written as a positive word in which $a$ appears as a prefix. There is also a suffix order, $\succcurlyeq$, defined by $a \succcurlyeq b$ if and only if $a b^{-1}$ is positive. These orders are known to be lattice orders, meaning that for every $a, b \in B_{n}$ there is a unique greatest common divisor $a \wedge b$ (resp. $a \wedge_{R} b$ ) and a unique least common multiple $a \vee b$ (resp. $\left.a \vee_{R} b\right)$ with respect to $\preccurlyeq($ resp. $\succcurlyeq)$.

The order $\preccurlyeq$ is, by definition, invariant under left-multiplication. That is, $a \preccurlyeq$ $b \Leftrightarrow c a \preccurlyeq c b$ for all $a, b, c \in B_{n}$. This implies that $c x \wedge c y=c(x \wedge y)$ and $c x \vee c y=c(x \vee y)$ for all $c, x, y \in B_{n}$. Similarly, $\succcurlyeq$ is invariant under rightmultiplication, and one has $x c \wedge_{R} y c=\left(x \wedge_{R} y\right) c$ and $x c \vee_{R} y c=\left(x \vee_{R} y\right) c$ for all $c, x, y \in B_{n}$. 
The braid group $B_{n}$ has a special element called Garside element or half twist,

$$
\Delta=\sigma_{1}\left(\sigma_{2} \sigma_{1}\right)\left(\sigma_{3} \sigma_{2} \sigma_{1}\right) \cdots\left(\sigma_{n-1} \cdots \sigma_{1}\right)
$$

Conjugation by $\Delta$ preserves $\preccurlyeq$ and $\succcurlyeq$. We denote by $\tau$ the inner automorphism of $B_{n}$ defined by $\Delta$, that is, $\tau(x)=\Delta^{-1} x \Delta$ for all $x \in B_{n}$. We recall that the center of $B_{n}$ is infinite cyclic, generated by $\Delta^{2}$. Hence $\tau$ preserves $\preccurlyeq$ and $\succcurlyeq$ (thus it preserves $\wedge, \vee, \wedge_{R}$ and $\vee_{R}$ ), and $\tau^{2}=\mathrm{id}$.

The set of positive prefixes of $\Delta$, denoted $[1, \Delta]=\left\{s \in B_{n} ; 1 \preccurlyeq s \preccurlyeq \Delta\right\}$, is called the set of simple elements of $B_{n}$. This set is finite, namely it has $n$ ! elements. Simple elements are the building blocks that conform the usual normal forms of braids. A simple element will be said to be proper if it is neither 1 nor $\Delta$.

The right complement $\partial(s)$ of a simple element $s$ is a simple element $t$ such that $s t=\Delta$, that is, $\partial(s)=s^{-1} \Delta$. The map $\partial$ is a bijection of the set of simple elements. Moreover, $\partial^{2}=\tau$. The left complement of a simple element is precisely $\partial^{-1}(s)=\Delta s^{-1}$. If a positive element is written as a product of two simple elements $s_{1} s_{2}$, we say that such a decomposition is left weighted if $s_{1}$ is the maximal simple prefix of $s_{1} s_{2}$, that is $s_{1} s_{2} \wedge \Delta=s_{1}$, or alternatively (multiplying from the left by $s_{1}^{-1}$ ), if $s_{2} \wedge \partial\left(s_{1}\right)=1$. We say that the decomposition $s_{1} s_{2}$ is right weighted if $s_{2}$ is the maximal simple suffix of $s_{1} s_{2}$, that is $s_{1} s_{2} \wedge_{R}$ $\Delta=s_{2}$, or alternatively (multiplying from the right by $s_{2}^{-1}$ ), if $s_{1} \wedge_{R} \partial^{-1}\left(s_{2}\right)=1$.

Given an element $x \in B_{n}$, we say that a decomposition $x=\Delta^{p} x_{1} \cdots x_{r}$ is the left normal form of $x$ if $p$ is the maximal integer such that $\Delta^{-p} x$ is positive, each $x_{i}$ is a proper simple element, and $x_{i} x_{i+1}$ is left weighted for $i=1, \ldots, r-1$. We say that a decomposition $x=x_{1}^{\prime} \cdots x_{r}^{\prime} \Delta^{p}$ is the right normal form of $x$ if $p$ is the maximal integer such that $x \Delta^{-p}$ is positive, each $x_{i}^{\prime}$ is a proper simple element, and $x_{i}^{\prime} x_{i+1}^{\prime}$ is right weighted for $i=1, \ldots, r-1$. The left and right normal forms are unique decompositions, and the numbers $p$ and $r$ are determined by $x$ and do not depend on the normal form (left or right) which is used to define them. In this way, one defines the infimum, supremum and canonical length of $x$ as, respectively, $\inf (x)=p, \sup (x)=p+r$ and $\ell(x)=r$.

It will be convenient for our purposes to use the following notation. When we deal with a positive element $x$, and we say that its left normal form is $x=x_{1} \cdots x_{r}$, (with no power of $\Delta$ on the left), we are allowing some of the initial factors to be equal to $\Delta$. That is, if $\inf (x)=p>0$, this will mean that $x_{1}=\cdots=x_{p}=\Delta$, so the actual normal form of $x$ would be $\Delta^{p} x_{p+1} \cdots x_{r}$.

There is still another normal form that we shall use. It is well known $[15,4]$ that, for every $x \in B_{n}$ there exist unique positive elements $u$ and $v$, with $u \wedge v=1$, such that $x=u^{-1} v$. If the left normal forms of $u$ and $v$ are, respectively, $u=u_{1} \cdots u_{r}$ and $v=v_{1} \cdots v_{s}$, the mixed normal form of $x$ is 
defined to be $x=u_{r}^{-1} \cdots u_{1}^{-1} v_{1} \cdots v_{s}$. We recall from [15] that, if $x$ can be written as $x=u^{-1} v$ with $u$ and $v$ positive elements with left normal forms $u_{1} \cdots u_{r}$ and $v_{1} \cdots v_{s}$, then $u_{r}^{-1} \cdots u_{1}^{-1} v_{1} \cdots v_{s}$ is the mixed normal form of $x$ if and only if $u_{1} \wedge v_{1}=1$.

We remark [15] that if $x=u_{r}^{-1} \cdots u_{1}^{-1} v_{1} \cdots v_{s}$ is in mixed normal form as above, the left normal form of $u^{-1}$ is $\Delta^{-r} u_{r}^{\prime} \cdots u_{1}^{\prime}$ (where $u_{i}^{\prime}=\partial^{-2 i-1}\left(u_{i}\right)$ ), and the left normal form of $x$ is equal to $x=\Delta^{-r} u_{r}^{\prime} \cdots u_{1}^{\prime} v_{1} \cdots v_{s}$. Therefore, from the mixed normal form one can already obtain $\inf (x)=-r, \ell(x)=r+s$ and hence $\sup (x)=s$.

The following technical results will be used later.

Lemma 2.1. Let $a$ and $b$ be positive braids whose left normal forms are $a=$ $a_{1} \cdots a_{r}, b=b_{1} \cdots b_{s}$, and whose right normal forms are $a=a_{1}^{\prime} \cdots a_{r}^{\prime}, b=$ $b_{1}^{\prime} \cdots b_{s}^{\prime}$. Consider $x=a^{-1} b$. If $\ell(x) \leq r+s-2 k+1$ for some integer $k>0$, then either $a_{1}^{\prime} \cdots a_{k}^{\prime} \preccurlyeq b_{1} \cdots b_{k}$ or $b_{1}^{\prime} \cdots b_{k}^{\prime} \preccurlyeq a_{1} \cdots a_{k}$.

Proof. Let $d=a \wedge b$, and write $a=d \alpha$ and $b=d \beta$. Then $x=a^{-1} b=\alpha^{-1} \beta$, where $\alpha$ and $\beta$ are positive elements such that $\alpha \wedge \beta=1$. Hence $\sup (\alpha)+\sup (\beta)+2 k-1=$ $\ell(x)+2 k-1 \leq r+s=\sup (a)+\sup (b)$. This implies that either $\sup (\alpha)+k \leq \sup (a)$ or $\sup (\beta)+k \leq \sup (b)$.

Suppose that $\sup (\alpha)+k \leq \sup (a)=r$. This means that $\alpha$ can be written as a product of at most $r-k$ simple elements. But $d \alpha=a=a_{1}^{\prime} \cdots a_{r}^{\prime}$, where the latter decomposition is in right normal form. It follows that $\alpha$ must be a suffix of $a_{k+1}^{\prime} \cdots a_{r}^{\prime}$, and then $a_{1}^{\prime} \cdots a_{k}^{\prime} \preccurlyeq d$. Hence $a_{1}^{\prime} \cdots a_{k}^{\prime} \preccurlyeq d \beta=b=b_{1} \cdots b_{s}$. Since the latter decomposition is in left normal form, one finally obtains $a_{1}^{\prime} \cdots a_{k}^{\prime} \preccurlyeq b_{1} \cdots b_{k}$. In the case $\sup (\beta)+k \leq \sup (b)=s$, one can apply the above reasoning to $\beta$ and $b$, to obtain $b_{1}^{\prime} \cdots b_{k}^{\prime} \preccurlyeq a_{1} \cdots a_{k}$.

Let us denote by $\varepsilon$ the automorphism of $B_{n}$ that sends $\sigma_{i}$ to $\sigma_{i}^{-1}$ for $i=1, \ldots, n-1$. Also, let rev: $B_{n} \rightarrow B_{n}$ be the anti-automorphism that sends each $\sigma_{i}$ to itself, that is, it sends a braid represented by a word $w$, to the braid represented by the same word written backwards. We will write, for every $x \in B_{n}, \operatorname{rev}(x)=\overleftarrow{x}$. Let us also denote inv: $B_{n} \rightarrow B_{n}$ the anti-automorphism $\operatorname{inv}(x)=x^{-1}$. Notice that the composition of any two of the maps in $\{\varepsilon$, rev, inv $\}$, in any order, yields the third one.

Lemma 2.2. Let $x$ be a positive braid with $\sup (x)=r+k$, where $r \geq k \geq 1$. If $\ell(\varepsilon(x) x) \leq 2 r+1$ then there exist positive braids $a$ and $b$ such that $x=\overleftarrow{a} b a$ and $\sup (b) \leq r$.

Proof. Let $x_{1} \cdots x_{r+k}$ be the left normal form of $x$ and let $y_{1} \cdots y_{r+k}$ be its right normal form. Hence $\overleftarrow{x_{r+k}} \cdots \overleftarrow{x_{1}}$ is the right normal form of $\overleftarrow{x}$ and $\overleftarrow{y_{r+k}} \cdots \overleftarrow{y_{1}}$ 
is its left normal form. Notice that $\varepsilon(x) x=(\overleftarrow{x})^{-1} x$. Hence, if $\ell(\varepsilon(x) x)=$ $\ell\left((\overleftarrow{x})^{-1} x\right) \leq 2 r+1$, Lemma 2.1 tells us that either $\overleftarrow{x_{r+k}} \cdots \overleftarrow{x_{r+1}} \preccurlyeq x_{1} \cdots x_{k}$ or $y_{1} \cdots y_{k} \preccurlyeq \overleftarrow{y_{r+k}} \cdots \overleftarrow{y_{r+1}}$.

Suppose that $\overleftarrow{x_{r+k}} \cdots \overleftarrow{x_{r+1}} \preccurlyeq x_{1} \cdots x_{k}$, and write $x_{1} \cdots x_{k}=\overleftarrow{x_{r+k}} \cdots \overleftarrow{x_{r+1}} \alpha$ for some positive $\alpha$. Since $r \geq k$, one can then write

$$
x=\left(\overleftarrow{x_{r+k}} \cdots \overleftarrow{x_{r+1}}\right) \alpha x_{k+1} \cdots x_{r}\left(x_{r+1} \cdots x_{r+k}\right)
$$

so the result follows in this case taking $a=x_{r+1} \cdots x_{r+k}$ and $b=\alpha x_{k+1} \cdots x_{r}$ (if $k=r$ then $b=\alpha$ ). Notice that $\sup (b) \leq r$ as $b$ is a suffix of $x_{1} \cdots x_{r}$.

Now suppose that $y_{1} \cdots y_{k} \preccurlyeq \overleftarrow{y_{r+k}} \cdots \overleftarrow{y_{r+1}}$, and write $\overleftarrow{y_{r+k}} \cdots \overleftarrow{y_{r+1}}=y_{1} \cdots y_{k} \beta$ for some positive $\beta$, which is equivalent to $y_{r+1} \cdots y_{r+k}=\overleftarrow{\beta} \overleftarrow{y_{k}} \cdots \overleftarrow{y_{1}}$. Then $x=\left(y_{1} \cdots y_{k}\right) y_{k+1} \cdots y_{r} \overleftarrow{\beta}\left(\overleftarrow{y_{k}} \cdots \overleftarrow{y_{1}}\right)$. Taking $a=\overleftarrow{y_{k}} \cdots \overleftarrow{y_{1}}$ and $b=y_{k+1} \cdots y_{r} \overleftarrow{\beta}$ which is a prefix of $y_{k+1} \cdots y_{k+r}$, the result follows also in this case.

We define a palindromic-free braid as a positive braid $x$ that cannot be decomposed as $x=\overleftarrow{a} b a$ for positive braids $a$ and $b$, where $a$ is nontrivial (see the equivalent definition 3.1). Palindromic-free braids will be crucial to show our main results. The above Lemma implies the following.

Corollary 2.3. Let $u$ be a positive braid with $\ell(x)=m$. Then $\ell(\varepsilon(x) x) \leq 2 m$. If moreover $u$ is palindromic-free and $m>1$, then $\ell(\varepsilon(x) x)=2 m$.

Proof. Recall that $\varepsilon(x)=(\overleftarrow{x})^{-1}$. Since the canonical length of a braid is preserved under reversing (by symmetry of the relations in $B_{n}$ ) and also under taking inverses (by [6]), it follows that $\ell(\varepsilon(x))=m$. Multiplying two braids of canonical length $m$ yields a braid of canonical length at most $2 m$, hence $\ell(\varepsilon(x) x) \leq 2 m$.

If $u$ is palindromic-free and $m>1$ we have the equality, as if we had $\ell(\varepsilon(x) x)<$ $2 m$, then by setting $r=m-1$ and $k=1$, we would have $\ell(\varepsilon(x) x) \leq 2 r+1$, which by Lemma 2.2 implies that $x$ is not palindromic-free.

\section{3. $\varepsilon$-TWISTED CONJUGACY AND PALINDROMIC-FREE BRAIDS}

Due to Theorem 1.3, the twisted conjugacy problem in $B_{n}$ will easily reduce to the $\varepsilon$-twisted conjugacy problem, namely given two braids $u, v \in B_{n}$ decide whether there exists another one $w \in B_{n}$ such that

$$
v=(\varepsilon(w))^{-1} u w .
$$

This problem has a very particular nature because $(\varepsilon(w))^{-1}=\overleftarrow{w}$, i.e. $\varepsilon$-twisted conjugating $u$ by $w$ amounts to multiply $u$ on the right by $w$ and on the left by $\overleftarrow{w}, v=\overleftarrow{w} u w$. Let us concentrate on this case, where the twisting is given by $\varepsilon$ 
Note that the $\varepsilon$-twisted conjugation of a positive braid by a positive braid yields a positive braid. Also, note that, for any braid $x \in B_{n}$ and any generator $\sigma_{i}, x$ and $\sigma_{i} x \sigma_{i}$ are $\varepsilon$-twisted conjugated. Imposing positivity, this yields to the following definition:

Definition 3.1. A positive braid $x$ is said to be palindromic-free if $\sigma_{i}^{-1} x \sigma_{i}^{-1}$ is not positive, for every $i=1, \ldots, n-1$.

In other words, if $x$ is a positive, palindromic-free braid and $\sigma_{i} \preccurlyeq x$, that is, $x=\sigma_{i} y$ for some positive $y$, then $y \nsucc \sigma_{i}$. However, notice that even if $x$ is palindromic-free, one may have simultaneously $\sigma_{i} \preccurlyeq x$ and $x \succcurlyeq \sigma_{i}$ for some $i$. For instance if $x=\sigma_{i}$, or if $x=\sigma_{i} \sigma_{j}$ with $|i-j| \geqslant 2$.

Proposition 3.2. Every braid $x \in B_{n}$ is $\varepsilon$-twisted conjugated to some positive, palindromic-free braid $y$.

Proof. It is well known that for every braid $x \in B_{n}$, the braid $x \Delta^{p}$ is positive for $p$ big enough. Since $\overleftarrow{\Delta}$ is positive (actually $\overleftarrow{\Delta}=\Delta$ ), it follows that $\overleftarrow{\Delta^{p}} x \Delta^{p}$ is positive for some $p$ big enough. Hence $x$ is $\varepsilon$-twisted conjugated to a positive braid $z$.

If $z$ is not palindromic-free, there will exist a letter $\sigma_{i}$ such that $z=\sigma_{i} z^{\prime} \sigma_{i}$ for some positive braid $z^{\prime}$ whose word length is smaller than that of $z$. And, since $\overleftarrow{\sigma_{i}}=\sigma_{i}, z$ is $\varepsilon$-twisted conjugated to $z^{\prime}$. Repeating this process, as the word length of the resulting braid decreases at each step, one finally obtains a palindromic-free positive braid $\varepsilon$-twisted conjugated to $z$, thus to $x$.

By the above argument, every positive braid $x$ has the form $x=\overleftarrow{c} y c$ for some positive, palindromic-free braid $y$. We remark that the element $y$ is not unique. For instance, if $x=\sigma_{2} \sigma_{1} \sigma_{2}=\sigma_{1} \sigma_{2} \sigma_{1}$, then $y$ could be equal to either $\sigma_{1}$ or $\sigma_{2}$. Another example is $x=\sigma_{3} \sigma_{2} \sigma_{1} \sigma_{2} \sigma_{3}=\sigma_{3} \sigma_{1} \sigma_{2} \sigma_{1} \sigma_{3}=\sigma_{1} \sigma_{2} \sigma_{3} \sigma_{2} \sigma_{1}$, so $y$ could be equal, in this case, to either $\sigma_{1}$ or $\sigma_{2}$ or $\sigma_{3}$.

Recall that we are trying to find an algorithm to solve the $\varepsilon$-twisted conjugacy problem in $B_{n}$. After the above discussion, one may think that a possible solution could be to compute the set of positive, palindromic-free braids, $\varepsilon$-twisted conjugated to a given one. Clearly, two braids $u$ and $v$ are $\varepsilon$-twisted conjugated if and only if their corresponding sets coincide. Unfortunately, this attempt does not work because the mentioned set is not always finite, as one can see in the following example.

Example 3.3. The set $\left\{\sigma_{3}^{n} \sigma_{2} \sigma_{3} \sigma_{4} \sigma_{5} \sigma_{1} \sigma_{2} \sigma_{3} \sigma_{4} \sigma_{1}^{n} ; n \geq 0\right\} \subset B_{6}$ is an infinite family of positive, palindromic-free braids, which are pairwise $\varepsilon$-twisted conjugated. 
Proof. We will show that for every $n \geq 0$ one has:

$$
\sigma_{5}^{n}\left(\sigma_{3}^{n} \sigma_{2} \sigma_{3} \sigma_{4} \sigma_{5} \sigma_{1} \sigma_{2} \sigma_{3} \sigma_{4} \sigma_{1}^{n}\right) \sigma_{5}^{n}=\left(\sigma_{1} \sigma_{3}\right)^{n} \sigma_{2} \sigma_{3} \sigma_{4} \sigma_{5} \sigma_{1} \sigma_{2} \sigma_{3} \sigma_{4}\left(\sigma_{3} \sigma_{1}\right)^{n} .
$$

So all braids in the above family are $\varepsilon$-twisted conjugated to $\sigma_{2} \sigma_{3} \sigma_{4} \sigma_{5} \sigma_{1} \sigma_{2} \sigma_{3} \sigma_{4}$, and so to each other. To see this, first notice that

$$
\begin{aligned}
\sigma_{5}\left(\sigma_{2} \sigma_{3} \sigma_{4} \sigma_{5} \sigma_{1} \sigma_{2} \sigma_{3} \sigma_{4}\right) & =\left(\sigma_{2} \sigma_{3}\right) \sigma_{5}\left(\sigma_{4} \sigma_{5} \sigma_{1} \sigma_{2} \sigma_{3} \sigma_{4}\right) \\
& =\left(\sigma_{2} \sigma_{3} \sigma_{4} \sigma_{5}\right) \sigma_{4}\left(\sigma_{1} \sigma_{2} \sigma_{3} \sigma_{4}\right) \\
& =\left(\sigma_{2} \sigma_{3} \sigma_{4} \sigma_{5} \sigma_{1} \sigma_{2}\right) \sigma_{4}\left(\sigma_{3} \sigma_{4}\right) \\
& =\left(\sigma_{2} \sigma_{3} \sigma_{4} \sigma_{5} \sigma_{1} \sigma_{2} \sigma_{3} \sigma_{4}\right) \sigma_{3} .
\end{aligned}
$$

On the other hand, by commutativity relations,

$$
\sigma_{2} \sigma_{3} \sigma_{4} \sigma_{5} \sigma_{1} \sigma_{2} \sigma_{3} \sigma_{4}=\sigma_{2} \sigma_{1} \sigma_{3} \sigma_{2} \sigma_{4} \sigma_{3} \sigma_{5} \sigma_{4}
$$

hence

$$
\begin{aligned}
\sigma_{1}\left(\sigma_{2} \sigma_{3} \sigma_{4} \sigma_{5} \sigma_{1} \sigma_{2} \sigma_{3} \sigma_{4}\right) & =\sigma_{1}\left(\sigma_{2} \sigma_{1} \sigma_{3} \sigma_{2} \sigma_{4} \sigma_{3} \sigma_{5} \sigma_{4}\right) \\
& =\left(\sigma_{2} \sigma_{1}\right) \sigma_{2}\left(\sigma_{3} \sigma_{2} \sigma_{4} \sigma_{3} \sigma_{5} \sigma_{4}\right) \\
& =\left(\sigma_{2} \sigma_{1} \sigma_{3} \sigma_{2}\right) \sigma_{3}\left(\sigma_{4} \sigma_{3} \sigma_{5} \sigma_{4}\right) \\
& =\left(\sigma_{2} \sigma_{1} \sigma_{3} \sigma_{2} \sigma_{4} \sigma_{3}\right) \sigma_{4}\left(\sigma_{5} \sigma_{4}\right) \\
& =\left(\sigma_{2} \sigma_{1} \sigma_{3} \sigma_{2} \sigma_{4} \sigma_{3} \sigma_{5} \sigma_{4}\right) \sigma_{5} \\
& =\left(\sigma_{2} \sigma_{3} \sigma_{4} \sigma_{5} \sigma_{1} \sigma_{2} \sigma_{3} \sigma_{4}\right) \sigma_{5}
\end{aligned}
$$

Therefore, as $\sigma_{1}, \sigma_{3}$ and $\sigma_{5}$ commute, one has

$$
\begin{aligned}
\sigma_{5}^{n} \sigma_{3}^{n}\left(\sigma_{2} \sigma_{3} \sigma_{4} \sigma_{5} \sigma_{1} \sigma_{2} \sigma_{3} \sigma_{4}\right) \sigma_{1}^{n} \sigma_{5}^{n} & =\sigma_{3}^{n}\left(\sigma_{2} \sigma_{3} \sigma_{4} \sigma_{5} \sigma_{1} \sigma_{2} \sigma_{3} \sigma_{4}\right) \sigma_{3}^{n} \sigma_{1}^{n} \sigma_{5}^{n} \\
& =\sigma_{1}^{n} \sigma_{3}^{n}\left(\sigma_{2} \sigma_{3} \sigma_{4} \sigma_{5} \sigma_{1} \sigma_{2} \sigma_{3} \sigma_{4}\right) \sigma_{3}^{n} \sigma_{1}^{n},
\end{aligned}
$$

and the claim follows.

It just remains to show that every element in the above family is palindromicfree. This could be easily done by using the standard topological representation of braids as collections of strands in $\mathbb{R}^{3}$, but we will show it algebraically.

If $n=0$, we have the braid $\alpha_{0}=\sigma_{2} \sigma_{3} \sigma_{4} \sigma_{5} \sigma_{1} \sigma_{2} \sigma_{3} \sigma_{4}$. We recall that the monoid $B_{6}^{+}$of positive braids embeds in $B_{6}$, so we just need to use positive relations from the standard presentation (1) to determine which generators are prefixes or suffixes of $\alpha_{0}$. But notice that in the above word, no matter how many commutativity relations we apply, we can never obtain a subword of the form $\sigma_{i} \sigma_{j} \sigma_{i}$, because between two appearances of the letter $\sigma_{i}$ one always has both $\sigma_{i-1}$ and $\sigma_{i+1}$. Hence, only commutativity relations can be applied, and it follows that this braid can only start with $\sigma_{2}$, and can only end with $\sigma_{4}$, thus it is palindromic-free. 
For $n>0$, the braid we are considering is $\alpha_{n}=\sigma_{3}^{n}\left(\sigma_{2} \sigma_{3} \sigma_{4} \sigma_{5} \sigma_{1} \sigma_{2} \sigma_{3} \sigma_{4}\right) \sigma_{1}^{n}$. Notice that:

$$
\begin{aligned}
\sigma_{3}\left(\sigma_{2} \sigma_{3} \sigma_{4} \sigma_{5} \sigma_{1} \sigma_{2} \sigma_{3} \sigma_{4}\right) & =\left(\sigma_{2} \sigma_{3}\right) \sigma_{2}\left(\sigma_{4} \sigma_{5} \sigma_{1} \sigma_{2} \sigma_{3} \sigma_{4}\right) \\
& =\left(\sigma_{2} \sigma_{3} \sigma_{4} \sigma_{5}\right) \sigma_{2}\left(\sigma_{1} \sigma_{2} \sigma_{3} \sigma_{4}\right) \\
& =\left(\sigma_{2} \sigma_{3} \sigma_{4} \sigma_{5} \sigma_{1} \sigma_{2}\right) \sigma_{1}\left(\sigma_{3} \sigma_{4}\right) \\
& =\left(\sigma_{2} \sigma_{3} \sigma_{4} \sigma_{5} \sigma_{1} \sigma_{2} \sigma_{3} \sigma_{4}\right) \sigma_{1}
\end{aligned}
$$

Hence $\alpha_{n}=\sigma_{3}^{2 n}\left(\sigma_{2} \sigma_{3} \sigma_{4} \sigma_{5} \sigma_{1} \sigma_{2} \sigma_{3} \sigma_{4}\right)$, and also $\alpha_{n}=\left(\sigma_{2} \sigma_{3} \sigma_{4} \sigma_{5} \sigma_{1} \sigma_{2} \sigma_{3} \sigma_{4}\right) \sigma_{1}^{2 n}$.

On one hand, the above two expressions of $\alpha_{n}$ show that it can start with $\sigma_{3}$ and also with $\sigma_{2}$. Suppose that it can also start with $\sigma_{1}$. As $\sigma_{3}^{2 n} \preccurlyeq \alpha_{n}$ and we are assuming that $\sigma_{1} \preccurlyeq \alpha_{n}$, it follows that $\sigma_{3}^{2 n} \vee \sigma_{1} \preccurlyeq \alpha_{n}$, that is $\sigma_{3}^{2 n} \sigma_{1} \preccurlyeq$ $\alpha_{n}$. Multiplying by $\sigma_{3}^{-2 n}$ from the left we obtain $\sigma_{1} \preccurlyeq \sigma_{2} \sigma_{3} \sigma_{4} \sigma_{5} \sigma_{1} \sigma_{2} \sigma_{3} \sigma_{4}$. But this is not possible as the latter braid can only start with $\sigma_{2}$. Hence $\sigma_{1} \npreceq \alpha_{n}$. Analogously, as $\sigma_{3}^{2 n} \vee \sigma_{4}=\sigma_{3}^{2 n} \sigma_{4} \sigma_{3}$, and also $\sigma_{3}^{2 n} \vee \sigma_{5}=\sigma_{3}^{2 n} \sigma_{5}$, it follows that $\sigma_{4} \npreceq \alpha_{n}$ and $\sigma_{5} \npreceq \alpha_{n}$. Therefore $\alpha_{n}$ can only start with either $\sigma_{2}$ or $\sigma_{3}$.

The symmetric argument shows that $\alpha_{n}$ can only end with either $\sigma_{1}$ or $\sigma_{4}$. Therefore $\alpha_{n}$ is palindromic-free, as we wanted to show.

Hence, the attempt to compute the set of all positive, palindromic-free braids, $\varepsilon$-twisted conjugated to a given one does not work. However, we shall save the idea by imposing a further condition which will assure the required finiteness of the set: we shall consider only elements with minimal canonical length. The set we will compute is then the following.

Definition 3.4. Given a braid $x \in B_{n}$, we define $M P F(x)$ to be the set of positive, palindromic-free braids, $\varepsilon$-twisted conjugated to $x$, of minimal canonical length.

Notice that if a positive braid $x$ is palindromic-free, then $\inf (x)=0, \operatorname{so} \sup (x)=$ $\ell(x)$. This gives us finiteness of $M P F(x)$ :

Proposition 3.5. For every $x \in B_{n}$, the set $M P F(x)$ is nonempty and finite, and it is an invariant of its $\varepsilon$-twisted conjugacy class.

Proof. $M P F(x)$ is an invariant of the $\varepsilon$-twisted conjugacy class of $x$ by definition. It is nonempty by Proposition 3.2, and it is finite since the set of elements of infimum zero, and given canonical length, is finite. 


\section{The twisted CONJugacy PRoBlem For $B_{n}$.}

In order to find a solution to the $\varepsilon$-twisted conjugacy problem in braid groups, we need a method to compute $M P F(x)$, given $x \in B_{n}$. For that purpose, we shall need the following technical results.

Lemma 4.1. Let $u$ be a positive, palindromic-free braid. Let $c$ be a positive braid with $\inf (c)=0$, whose left normal form is $c=c_{1} \cdots c_{s}$. Denote $k_{i}=$ $\inf \left(\overleftarrow{c_{i}} \cdots \overleftarrow{c_{1}} u c_{1} \cdots c_{i}\right)$. Then $k_{i+1} \leq k_{i}+1$ for $i=0, \ldots, s-1$. In particular $\inf \left(\overleftarrow{c_{i}} \cdots \overleftarrow{c_{1}} u c_{1} \cdots c_{i}\right) \leq i$ for $i=0, \ldots, s$

Proof. Recall that, since $u$ is palindromic-free, $\inf (u)=0$. As the infimum of an element can increase by at most one when multiplied by a simple element, one has either $\inf \left(u c_{1}\right)=0$ or $\inf \left(u c_{1}\right)=1$.

Suppose that $\inf \left(u c_{1}\right)=0$, that is, $\Delta$ is not a prefix of $u c_{1}$. It is well known that, as $c_{1} \cdots c_{s}$ is in left normal form, then $\inf \left(u c_{1} \cdots c_{s}\right)=0$. Since the infimum of an element can increase by at most one when it is multiplied by a simple element, one has $\inf \left(\overleftarrow{c_{i}} \cdots \overleftarrow{c_{1}} u c_{1} \cdots c_{s}\right) \leq i$, moreover $\inf \left(\overleftarrow{c_{i}} \cdots \overleftarrow{c_{1}} u c_{1} \cdots c_{s}\right) \leq$ $\inf \left(\overleftarrow{c_{i-1}} \cdots \overleftarrow{c_{1}} u c_{1} \cdots c_{s}\right)+1$ for $i=1, \ldots, s$. It suffices then to show that $k_{i}=\inf \left(\overleftarrow{c_{i}} \cdots \overleftarrow{c_{1}} u c_{1} \cdots c_{s}\right)$ for $i=0, \ldots, s$. But since we already showed that $\inf \left(\overleftarrow{c_{i}} \cdots \overleftarrow{c_{1}} u c_{1} \cdots c_{s}\right) \leq i$, and $c_{1} \cdots c_{s}$ is in left normal form, then $\Delta^{p}$ is a prefix of $\overleftarrow{c_{i}} \cdots \overleftarrow{c_{1}} u c_{1} \cdots c_{s}$ (necessarily $p \leq i$ ) if and only if it is a prefix of $\overleftarrow{c_{i}} \cdots \overleftarrow{c_{1}} u c_{1} \cdots c_{i}$. Hence $\inf \left(\overleftarrow{c_{i}} \cdots \overleftarrow{c_{1}} u c_{1} \cdots c_{s}\right)=\inf \left(\overleftarrow{c_{i}} \cdots \overleftarrow{c_{1}} u c_{1} \cdots c_{i}\right)=k_{i}$ for $i=0, \ldots, s$, as we wanted to show.

Now suppose that $\inf \left(u c_{1}\right)=1$, that is, $u c_{1}=v \Delta$ for some positive $v$, prefix of $u$. This means that $u=v \partial^{-1}\left(c_{1}\right)$. Since $u$ is palindromic-free, one has $\overleftarrow{\partial^{-1}\left(c_{1}\right)} \wedge v=1$. But it is easy to see that $\overleftarrow{\partial^{-1}\left(c_{1}\right)}=\partial\left(\overleftarrow{c_{1}}\right)$, so one has $\partial\left(\overleftarrow{c_{1}}\right) \wedge v=1$, that is, the decomposition $\overleftarrow{c_{1}} v$ is left-weighted as written. This in particular implies that $\inf \left(\overleftarrow{c_{1}} v\right)=0$ and, since $\overleftarrow{c_{s}} \cdots \overleftarrow{c_{1}}$ is in right normal form, that $\inf \left(\overleftarrow{c_{s}} \cdots \overleftarrow{c_{1}} v\right)=0$. Hence $\inf \left(\overleftarrow{c_{s}} \ldots \overleftarrow{c_{1}} u c_{1}\right)=\inf \left(\overleftarrow{c_{s}} \cdots \overleftarrow{c_{1}} v \Delta\right)=1=$ $k_{1}=k_{0}+1$. As the infimum can increase by at most one when an element is multiplied by a simple one, then one has $\inf \left(\overleftarrow{c_{s}} \cdots \overleftarrow{c_{1}} u c_{1} \cdots c_{i}\right) \leq i$, moreover $\inf \left(\overleftarrow{c_{s}} \cdots \overleftarrow{c_{1}} u c_{1} \cdots c_{i}\right) \leq \inf \left(\overleftarrow{c_{s}} \cdots \overleftarrow{c_{1}} u c_{1} \cdots c_{i-1}\right)+1$ for $i=1, \ldots, s$. Repeating the argument of the previous case, one has $k_{i}=\inf \left(\overleftarrow{c_{s}} \cdots \overleftarrow{c_{1}} u c_{1} \cdots c_{i}\right)$ for $i=0, \ldots, s$, and the result is shown.

Corollary 4.2. Let $u$ be a positive, palindromic-free braid. Let c be a positive braid with $\inf (c)=0$, and whose left normal form is $c=c_{1} \cdots c_{s}$. If $\inf \left(\overleftarrow{c_{s}} \cdots \overleftarrow{c_{1}} u c_{1} \cdots c_{s}\right)=s$, then $\inf \left(\overleftarrow{c_{i}} \cdots \overleftarrow{c_{1}} u c_{1} \cdots c_{i}\right)=i$ for $i=0, \ldots, s$

Proof. Let $k_{i}=\inf \left(\overleftarrow{c_{i}} \cdots \overleftarrow{c_{1}} u c_{1} \cdots c_{i}\right)$ for $i=0, \ldots, s$. We know that $k_{0}=0$ since $u$ is palindromic-free, and that $k_{i+1} \leq k_{i}+1$ by the previous result. By induction 
on $s$, it follows that $k_{s} \leq s$ and the equality holds if and only if $k_{i+1}=k_{i}+1$ for $i=0, \ldots, s-1$. But we have $k_{s}=s$ by hypothesis, hence $k_{i}=i$ for $i=0, \ldots, s$, as we wanted to show.

Corollary 4.3. Let $u, v \in B_{n}$ be positive, palindromic-free braids. Let $a$ and $b$ be nontrivial positive braids such that $a \wedge_{R} b=1$ (hence $\left.\inf (a)=\inf (b)=0\right)$. Suppose that $\overleftarrow{a} u a=\overleftarrow{b} v b$. Then $\ell(a)=\ell(b)$

Proof. Denote $\ell(a)=p$ and $\ell(b)=q$, and write $a=a_{1} \cdots a_{p}$ and $b=b_{1} \cdots b_{q}$ in right normal forms. Consider $b^{*}=b^{-1} \Delta^{q}$. Then $b^{*}$ is a positive braid with $\inf \left(b^{*}\right)=0$. Namely, its right normal form is $b^{*}=\partial\left(b_{q}\right) \partial^{3}\left(b_{q-1}\right) \cdots \partial^{2 q-1}\left(b_{1}\right)$. Then consider the product

$$
a b^{*}=a_{1} \cdots a_{p} \partial\left(b_{q}\right) \partial^{3}\left(b_{q-1}\right) \cdots \partial^{2 q-1}\left(b_{1}\right) .
$$

We claim that the above decomposition is the right normal form of $a b^{*}$. We just need to show that $a_{p} \partial\left(b_{q}\right)$ is right-weighted as written. But $a \wedge_{R} b=1$, so $1=a_{p} \wedge_{R} b_{q}=a_{p} \wedge_{R} \partial^{-1}\left(\partial\left(b_{q}\right)\right)$, which precisely means that $a_{p} \partial\left(b_{q}\right)$ is rightweighted, showing the claim. This implies in particular that $\inf \left(a b^{*}\right)=0$ and $\ell\left(a b^{*}\right)=p+q$.

Notice that $\overleftarrow{b^{*}} \overleftarrow{a} u a b^{*}=\overleftarrow{b^{*}} \overleftarrow{b} v b b^{*}=\Delta^{q} v \Delta^{q}$. Since $\inf (v)=0$ as $v$ is palindromic free, one has $\inf \left(\overleftarrow{b^{*}} \overleftarrow{a} u a b^{*}\right)=\inf \left(\Delta^{q} v \Delta^{q}\right)=2 q$. On the other hand, $\inf \left(a b^{*}\right)=0$ and $\ell\left(a b^{*}\right)=p+q$, so Lemma 4.1 implies that $\inf \left(\overleftarrow{b^{*}} \overleftarrow{a} u a b^{*}\right) \leq p+q$. Therefore $2 q \leq p+q$, that is, $q \leq p$. By symmetry, one also has $p \leq q$, so the equality holds.

Recall that we want to find a method to compute, for any given braid $x \in B_{n}$, the set $M P F(x)$ i.e. the (finite) set of positive, palindromic-free, $\varepsilon$-twisted conjugates of $x$ of minimal canonical length. Notice that if two elements $u$ and $v$ are $\varepsilon$ twisted conjugated, that is, if $\overleftarrow{c} u c=v$ for some braid $c$, then we can multiply on both sides by a suitable power of $\Delta$ such that $c \Delta^{p}$ is positive, in such a way that $\Delta^{p \overleftarrow{c}} u c \Delta^{p}=\Delta^{p} v \Delta^{p}$, so we have written $\overleftarrow{A} u A=\overleftarrow{B} v B$ with $A$ and $B$ positive. Moreover, if $d=A \wedge_{R} B$ is the maximal common suffix of $A$ and $B$, then multiplying the above equality from the right by $d^{-1}$ and from the left by $(\overleftarrow{d})^{-1}$, we finally get $\overleftarrow{a} u a=\overleftarrow{b} v b$, with $a$ and $b$ positive and such that $a \wedge_{R} b=1$ as in the hypothesis of the above result. We will be specially interested in the case in which $a$ and $b$ are simple elements.

Definition 4.4. We will say that two elements $u, v \in B_{n}$ are simply $\varepsilon$-twisted conjugated, or that they are related by a simple $\varepsilon$-twisted conjugation, if there exist simple elements $a$ and $b$ such that $\overleftarrow{a} u a=\overleftarrow{b} v b$ 
The main result of this section is analogous, with respect to $\varepsilon$-twisted conjugacy, to the following famous result by El-Rifai and Morton with respect to conjugacy.

Theorem 4.5. [6] Let $u, v \in B_{n}$ be conjugated braids such that $\ell(u) \leq r$ and $\ell(v) \leq r$ for some $r$. Then there is a chain $u=u_{0}, u_{1}, \ldots, u_{k}=v$, with $\ell\left(u_{i}\right) \leq r$ for all $i$, such that $u_{i-1}$ is conjugated to $u_{i}$ by a simple element, for $i=1, \ldots, k$. Namely, if $c$ is a positive element such that $c^{-1} u c=v$, and $c=c_{1} \cdots c_{k}$ is its left normal form, then one can take $u_{i}=c_{i}^{-1} \cdots c_{1}^{-1} u c_{1} \cdots c_{i}$.

In our case, dealing with $\varepsilon$-twisted conjugacy, we will restrict to positive, palindromic-free braids.

Theorem 4.6. Let $u, v \in B_{n}$ be positive, palindromic-free, $\varepsilon$-twisted conjugated braids such that $\ell(u) \leq r$ and $\ell(v) \leq r$ for some $r$. Then there is a chain $u=u_{0}, u_{1}, \ldots, u_{k}=v$ of positive, palindromic-free braids, with $\ell\left(u_{i}\right) \leq r$ for all $i$, such that $u_{i-1}$ is simply $\varepsilon$-twisted conjugated to $u_{i}$, for $i=1, \ldots, k$.

Proof. As we saw above, there are positive elements $a$ and $b$, with $a \wedge_{R} b=1$, such that $\overleftarrow{a} u a=\overleftarrow{b} v b$. Since $u$ and $v$ are palindromic free, $a$ is trivial if and only if so is $b$. If $a$ and $b$ are nontrivial, the hypotheses of Corollary 4.3 are satisfied, thus $\ell(a)=\ell(b)=p$ in any case. We will show the result by induction on $p$. If $p=0$ the result is trivially true, so we will assume that $p>0$ and that the result is true for all values between 0 and $p-1$.

The strategy of the proof will be to find some palindromic-free braid $w$ with $\ell(w) \leq r$, such that $\overleftarrow{s} u s=\overleftarrow{t} w t$ for some simple braids $s$ and $t$ (this is a chain of length 1 from $u$ to $w$ ), and also $\overleftarrow{y} w y=\overleftarrow{z} v z$ for some positive elements $y, z$ such that $y \wedge_{R} z=1$ and $\ell(y)=\ell(z) \leq p-1$. The induction hypothesis provides a chain from $w$ to $v$, so the result will follow by concatenating both chains.

We start as in the proof of Corollary 4.3, defining $b^{*}=b^{-1} \Delta^{p}$, and noticing that $\inf \left(a b^{*}\right)=0, \ell\left(a b^{*}\right)=2 p$ and $\overleftarrow{b^{*}} \overleftarrow{a} u a b^{*}=\Delta^{p} v \Delta^{p}$. Denote $c=a b^{*}$, and let $c=c_{1} \cdots c_{2 p}$ be its left normal form. Then $\overleftarrow{c} u c=\Delta^{p} v \Delta^{p}$. Since $v$ is palindromic-free, thus $\inf (v)=0$, one has $\inf (\overleftarrow{c} u c)=2 p$. By Corollary 4.2 one has $\inf \left(\overleftarrow{c_{i}} \cdots \overleftarrow{c_{1}} u c_{1} \cdots c_{i}\right)=i$ for $i=1, \ldots, 2 p$. In particular $\inf \left(\overleftarrow{c_{2}} \overleftarrow{c_{1}} u c_{1} c_{2}\right)=2$ hence $\overleftarrow{c_{2}} \overleftarrow{c_{1}} u c_{1} c_{2}=\Delta w^{\prime} \Delta$ for some positive braid $w^{\prime}$

Multiplying the above equality on the right by $c_{2}^{-1}$ and on the left by its reverse, we obtain $\overleftarrow{c_{1}} u c_{1}=\overleftarrow{\partial^{-1}\left(c_{2}\right)} w^{\prime} \partial^{-1}\left(c_{2}\right)$. Hence $u$ and $w^{\prime}$ are simply $\varepsilon$-twisted conjugated. But $w^{\prime}$ is not necessarily palindromic-free, and one does not necessarily have $\ell\left(w^{\prime}\right) \leq r$. Let us see that we can replace $w^{\prime}$ by some $w$ that satisfies the required hypothesis.

Recall that $\overleftarrow{c_{2}} \overleftarrow{c_{1}} u c_{1} c_{2}=\Delta w^{\prime} \Delta$. Since the left hand side is a product of at most $r+4$ simple elements, it follows that $\sup \left(w^{\prime}\right) \leq r+2$. Moreover, multiplying each 
side of the equality, from the left, by its image under $\varepsilon$, one has

$$
\left(\varepsilon\left(\overleftarrow{c_{2}} \overleftarrow{c_{1}} u c_{1} c_{2}\right)\right)\left(\overleftarrow{c_{2}} \overleftarrow{c_{1}} u c_{1} c_{2}\right)=\left(\varepsilon\left(\Delta w^{\prime} \Delta\right)\right)\left(\Delta w^{\prime} \Delta\right)
$$

Hence:

$$
\left(c_{2}^{-1} c_{1}^{-1} \varepsilon(u) \varepsilon\left(c_{1}\right) \varepsilon\left(c_{2}\right)\right)\left(\overleftarrow{c_{2}} \overleftarrow{c_{1}} u c_{1} c_{2}\right)=\left(\Delta^{-1} \varepsilon\left(w^{\prime}\right) \Delta^{-1}\right)\left(\Delta w^{\prime} \Delta\right)
$$

Since $\varepsilon\left(c_{i}\right)=\left(\overleftarrow{c_{i}}\right)^{-1}$, one obtains:

$$
c_{2}^{-1} c_{1}^{-1}(\varepsilon(u) u) c_{1} c_{2}=\tau\left(\varepsilon\left(w^{\prime}\right) w^{\prime}\right) .
$$

In the same way, from the equality

$$
\left(\overleftarrow{c_{2 p}} \cdots \overleftarrow{c_{1}}\right) u\left(c_{1} \cdots c_{2 p}\right)=\Delta^{p} v \Delta^{p}
$$

one gets:

$$
\left(c_{2 p}^{-1} \cdots c_{1}^{-1}\right) \varepsilon(u) u\left(c_{1} \cdots c_{2 p}\right)=\tau^{p}(\varepsilon(v) v) .
$$

Recall that $\ell(u) \leq r$ and $\ell(v) \leq r$, so by Corollary 2.3 one has $\ell(\varepsilon(u) u) \leq$ $2 r$ and $\ell(\varepsilon(v) v) \leq 2 r$, thus $\ell\left(\tau^{p}(\varepsilon(v) v)\right) \leq 2 r$. Therefore, by Theorem 4.5, $\ell\left(\tau\left(\varepsilon\left(w^{\prime}\right) w^{\prime}\right)\right)=\ell\left(c_{2}^{-1} c_{1}^{-1} \varepsilon(u) u c_{1} c_{2}\right) \leq 2 r$. Hence $\ell\left(\varepsilon\left(w^{\prime}\right) w^{\prime}\right) \leq 2 r$.

We claim that there are positive braids $x$ and $w$, such that $w^{\prime}=\overleftarrow{x} w x$ and $\sup (w) \leq r$. First, if $\sup \left(w^{\prime}\right) \leq r$ one can take $x=1$ and $w=w^{\prime}$. Second, if $\sup \left(w^{\prime}\right)=r+1$, notice that $r \geq 1$ and $\ell\left(\varepsilon\left(w^{\prime}\right) w^{\prime}\right) \leq 2 r$, so the claim follows from Lemma 2.2, taking $k=1$. We must then show the claim in the case $\sup \left(w^{\prime}\right)=r+2$.

Suppose that $\sup \left(w^{\prime}\right)=r+2$, and recall that $\ell\left(\varepsilon\left(w^{\prime}\right) w^{\prime}\right) \leq 2 r=2 \sup \left(w^{\prime}\right)-4$. If $\sup \left(w^{\prime}\right) \geq 4$, the claim follows from Lemma 2.2, taking $k=2$. Therefore the only remaining case is $\sup \left(w^{\prime}\right)=3, r=1$ and $\ell\left(\varepsilon\left(w^{\prime}\right) w^{\prime}\right) \leq 2$. Let $d=w^{\prime} \wedge \operatorname{rev}\left(w^{\prime}\right)$ and write $w^{\prime}=d \alpha$ and $\operatorname{rev}\left(w^{\prime}\right)=d \beta$. Notice that $\varepsilon\left(w^{\prime}\right) w^{\prime}=\operatorname{rev}\left(w^{\prime}\right)^{-1} w^{\prime}=\beta^{-1} d^{-1} d \alpha$, hence the mixed normal form of $\varepsilon\left(w^{\prime}\right) w^{\prime}$ is precisely $\beta^{-1} \alpha$. Moreover, since the word length of $w^{\prime}$ and $\operatorname{rev}\left(w^{\prime}\right)$ coincide, one has $\alpha=1$ if and only if $\beta=1$. Hence, $\operatorname{since} \sup (\alpha)+\sup (\beta)=\ell\left(\varepsilon\left(w^{\prime}\right) w^{\prime}\right) \leq 2$, one must necessarily have either $\sup (\alpha)=\sup (\beta)=0$ or $\sup (\alpha)=\sup (\beta)=1$, that is, $\alpha$ and $\beta$ are (possibly trivial) simple elements.

Write $w^{\prime}=a_{1} a_{2} a_{3}$ in left normal form. The right normal form of $\operatorname{rev}\left(w^{\prime}\right)$ is then $\overleftarrow{a_{3}} \overleftarrow{a_{2}} \overleftarrow{a_{1}}$. Since $\operatorname{rev}\left(w^{\prime}\right)=d \beta$ and $\beta$ is simple, it follows that $\overleftarrow{a_{3}} \overleftarrow{a_{2}} \preccurlyeq d$, hence $\overleftarrow{a_{3}} \overleftarrow{a_{2}} \preccurlyeq d \alpha=w^{\prime}=a_{1} a_{2} a_{3}$. Since the latter decomposition is in left normal form, one has $\overleftarrow{a_{3}} \overleftarrow{a_{2}} \preccurlyeq a_{1} a_{2}$, and also $\overleftarrow{a_{3}} \preccurlyeq a_{1}$. Write then $w^{\prime}=\overleftarrow{a_{3}}\left(c a_{2}\right) a_{3}$ for some positive $c$. Now if $c a_{2}$ is simple we are done, as one can take $x=a_{3}$ and $w=c a_{2}$. Otherwise, write $c a_{2}=b_{1} b_{2}$ in left normal form, and recall that $\overleftarrow{a_{3}} \overleftarrow{a_{2}} \preccurlyeq a_{1} a_{2}$, so $\overleftarrow{a_{2}} \preccurlyeq c a_{2}=b_{1} b_{2}$. Then $\overleftarrow{a_{2}} \preccurlyeq b_{1}$. On the other hand, since $c a_{2}=b_{1} b_{2}$ and the latter decomposition is left weighted, one has $a_{2} \succcurlyeq b_{2}$ and then $\overleftarrow{b_{2}} \preccurlyeq \overleftarrow{a_{2}}$. Concatenating the last two inequalities, one finally obtains $\overleftarrow{b_{2}} \preccurlyeq \overleftarrow{a_{2}} \preccurlyeq b_{1}$. Therefore one can 
write $b_{1}=\overleftarrow{b_{2}} w$ for some simple element $w$, and one has $w^{\prime}=\left(\overleftarrow{a_{3}} \overleftarrow{b_{2}}\right) w\left(b_{2} a_{3}\right)$ Taking $x=b_{2} a_{3}$, the claim is shown.

Notice that if $w$ is not palindromic-free, we can still decompose $w=\overleftarrow{y} w^{\prime \prime} y$ where $y$ is positive and $w^{\prime \prime}$ is palindromic-free. Moreover, $\sup \left(w^{\prime \prime}\right) \leq \sup (w) \leq r$. Therefore, replacing $x$ by $y x$ and $w$ by $w^{\prime \prime}$ if necessary, we can assume that $w^{\prime}=\overleftarrow{x} w x$, where $x$ is positive and $w$ is palindromic-free with $\sup (w) \leq r$

Now recall that $\overleftarrow{c_{1}} u c_{1}=\overleftarrow{\partial^{-1}\left(c_{2}\right)} w^{\prime} \partial^{-1}\left(c_{2}\right)$ for simple elements $c_{1}$ and $c_{2}$. By the above claim, $\overleftarrow{c_{1}} u c_{1}=\left(\overleftarrow{\partial^{-1}\left(c_{2}\right)} \overleftarrow{x}\right) w\left(x \partial^{-1}\left(c_{2}\right)\right)$. Multiplying this equality from the right by $\left(c_{1} \wedge_{R}\left(x \partial^{-1}\left(c_{2}\right)\right)\right)^{-1}$ and from the left by its reverse, we obtain $\overleftarrow{s} u s=\overleftarrow{t} w t$ for positive braids $s$ and $t$ such that $s \wedge_{R} t=1$. Now $s$ is simple as it is a prefix of $c_{1}$, hence $t$ is simple by Corollary 4.3. Therefore $u$ and $w$ are simply $\varepsilon$-twisted conjugated, positive, palindromic-free braids, whose canonical length is at most $r$. This is the first step of our required chain.

Now notice that

$$
\begin{aligned}
\overleftarrow{c_{2 p}} \cdots \overleftarrow{c_{3}} \Delta w^{\prime} \Delta c_{3} \cdots c_{2 p} & =\overleftarrow{c_{2 p}} \cdots \overleftarrow{c_{3}} \overleftarrow{c_{2}} \overleftarrow{\partial^{-1}\left(c_{2}\right)} w^{\prime} \partial^{-1}\left(c_{2}\right) c_{2} c_{3} \cdots c_{2 p} \\
& =\overleftarrow{c_{2 p}} \cdots \overleftarrow{c_{2}} \overleftarrow{c_{1}} u c_{1} c_{2} \cdots c_{2 p} \\
& =\Delta^{p} v \Delta^{p}
\end{aligned}
$$

Hence

$$
\tau^{-1}\left(\overleftarrow{c_{2 p}} \cdots \overleftarrow{c_{3}}\right) w^{\prime} \tau^{-1}\left(c_{3} \cdots c_{2 p}\right)=\Delta^{p-1} v \Delta^{p-1}
$$

For simplicity, we will denote $d_{i}=\tau^{-1}\left(c_{i+2}\right)$ for $i=1, \ldots, 2 p-2$. Hence we have:

$$
\left(\overleftarrow{d_{2 p-2}} \cdots \overleftarrow{d_{1}}\right) w^{\prime}\left(d_{1} \cdots d_{2 p-2}\right)=\Delta^{p-1} v \Delta^{p-1}
$$

Recalling that $w^{\prime}=\overleftarrow{x} w x$, and multiplying the above equality from the right by $\left(d_{p} \cdots d_{2 p-2}\right)^{-1}$ and from the left by its reverse, we finally obtain:

$$
\left(\overleftarrow{d_{p-1}} \cdots \overleftarrow{d_{1}} \overleftarrow{x}\right) w\left(x d_{1} \cdots d_{p-1}\right)=\left(\overleftarrow{e_{p-1}} \cdots \overleftarrow{e_{1}}\right) v\left(e_{1} \cdots e_{p-1}\right)
$$

where $e_{1}, \ldots, e_{p-1}$ are simple elements and $e_{1} \cdots e_{p-1}=\Delta^{p-1}\left(d_{p} \cdots d_{2 p-2}\right)^{-1}$. Reducing the above equality, if necessary, by the biggest common suffix of $\left(x d_{1} \cdots d_{p-1}\right)$ and $\left(e_{1} \cdots e_{p-1}\right)$, it follows that there exist positive braids $y$ and $z$ such that $y \wedge_{R} z=1$, and $\overleftarrow{y} w y=\overleftarrow{z} v z$. Recall that $w$ and $v$ are palindromicfree and, by Corollary 4.3 and as $z$ is a prefix of $e_{1} \cdots e_{p-1}, \ell(y)=\ell(z) \leq p-1$. Therefore the induction hypothesis provides the remaining part of the required chain, and the result is shown.

Corollary 4.7. Let $u, v \in B_{n}$ be positive, palindromic-free, $\varepsilon$-twisted conjugated braids of minimal canonical length in their $\varepsilon$-twisted conjugacy class, say $r=$ 
$\ell(u)=\ell(v)$. Then there is a chain $u=u_{0}, u_{1}, \ldots, u_{k}=v$ of positive, palindromicfree braids, with canonical length $\ell\left(u_{i}\right)=r$ for all $i$, such that $u_{i-1}$ is simply $\varepsilon$-twisted conjugated to $u_{i}$, for $i=1, \ldots, k$.

Corollary 4.8. There exists an algorithm to compute $M P F(x)$ for any given braid $x \in B_{n}$.

Proof. If $x=1$ then $\operatorname{MPF}(x)=\{1\}$. So, let us assume $x \neq 1$.

First of all, compute a positive, palindromic-free, $\varepsilon$-twisted conjugate of $x$, say $y$, as it is explained in Proposition 3.2. Let $r=\ell(y) \geqslant 1$, and let $S=\{y\} \subset B_{n}$.

Now, consider the following operation, which will have to be subsequently applied until all elements in $S$ have been processed:

Choose $z \in S$ which has not been processed, compute all positive palindromic-free elements which are simply $\varepsilon$-twisted conjugated to $z$ and have canonical length less than or equal to $r$ (this is clearly a finite, computable set), and then do the following: 1) if one of them, say $z^{\prime}$, has length less than $r$, kill the whole process, reset $y=z^{\prime}, S=\left\{z^{\prime}\right\}, r=\ell\left(z^{\prime}\right)$ and start the algorithm again; 2) otherwise, add to $S$ all the computed elements (which have canonical length exactly equal to $r$ ), and mark $z$ as processed.

At each application of such operation, either the set $S$ gets restarted and $r$ strictly decreased, or the set $S$ gets increased by the addition of the new elements computed (some of which could already be present in the former $S$ ). But $r \geqslant 1$ can only decrease a finite number of times, and $|S|$ can only increase a finite number of times, since the number of braids with infimum zero and given canonical length is finite (recall that palindromic-free elements have infimum zero).

Hence, after a finite number of applications of the previous operation (running over all elements $z \in S$ ), we shall get a set $S \neq \emptyset$ closed under this operation, i.e. such that when applying that operation to any $z \in S$ the set neither gets restarted nor gets increased (that is, all the elements computed are already present in $S$ ). At this time, Theorem 4.6 implies that the canonical length of the elements in $S$ (which is constant) is the smallest possible among all positive palindromic-free braids which are $\varepsilon$-twisted conjugated to $x$. That is, $S \subseteq M P F(x)$.

Now, let $u \in M P F(x)$. Choosing an arbitrary $v \in S$, Corollary 4.7 tells us that $u$ and $v$ are connected by a chain of positive, palindromic-free braids of minimal canonical length, each simply $\varepsilon$-twisted conjugated to the following one. Hence, by construction of $S$, we have $u \in S$. Therefore, $S=M P F(x)$.

Theorem 4.9. The twisted conjugacy problem is solvable in the braid group $B_{n}$. 
Proof. Suppose we are given an automorphism $\varphi: B_{n} \rightarrow B_{n}$ (by the images of the generators), and two braids $u, v \in B_{n}$. We have to decide whether there exists $x \in B_{n}$ such that $v=(\varphi(x))^{-1} u x$, and in the positive case compute such an $x$.

By Theorem 1.3, either $\varphi$ is a conjugation $\left(\varphi=\gamma_{w}\right.$ for some $\left.w \in B_{n}\right)$, or it is $\varepsilon$ followed by a conjugation $\left(\varphi=\gamma_{w} \varepsilon\right.$ for some $\left.w \in B_{n}\right)$. We can clearly make this decision effective, and compute such a $w$. Indeed, in order to check whether $\varphi=$ $\gamma_{w}$, we need to find some braid $w$ such that $w^{-1} \sigma_{i} w=\varphi\left(\sigma_{i}\right)$ for $i=1, \ldots, n-1$. This is an instance of the so-called multiple simultaneous conjugacy problem in $B_{n}$, and algorithms to solve it (and to find such $w$ ) can be found in $[12,11]$. On the other hand, checking whether $\varphi=\gamma_{w} \varepsilon$ and finding such $w$ reduces to solving another instance of the multiple simultaneous conjugacy problem in $B_{n}$ : namely, it amounts to find $w$ such that $w^{-1} \sigma_{i}^{-1} w=\varphi\left(\sigma_{i}\right)$ for $i=1, \ldots, n-1$. (Alternatively, in our specific situation, we can make the following conceptually much easier brute force algorithm: knowing, by Theorem 1.3, that there exists $w \in B_{n}$ such that either $w^{-1} \sigma_{i} w=\varphi\left(\sigma_{i}\right)$ for $i=1, \ldots, n-1$, or $w^{-1} \sigma_{i}^{-1} w=\varphi\left(\sigma_{i}\right)$ for $i=1, \ldots, n-1$, one can always enumerate all words $w \in B_{n}$ and keep checking both conditions until finding the good one with the correct $w$.) We can therefore assume that $w$ is known, and that $\varphi$ is equal either to $\gamma_{w}$ or to $\gamma_{w} \varepsilon$.

In the first case $\varphi(x)=w^{-1} x w$, and the equation $v=(\varphi(x))^{-1} u x$ is equivalent to $w v=x^{-1}(w u) x$. Deciding the existence of such an $x$ and finding it, is just an instance of the standard conjugacy problem in $B_{n}$ (applied to $w v$ and $w u$ ), which is well-known to be solvable, see Theorem 1.2.

In the second case, $\varphi(x)=w^{-1} \varepsilon(x) w$, and the equation $v=(\varphi(x))^{-1} u x$ is equivalent to $w v=(\varepsilon(x))^{-1}(w u) x=\overleftarrow{x}(w u) x$. Deciding the existence of such an $x$ and finding it, is an instance of the $\varepsilon$-twisted conjugacy problem in $B_{n}$ (applied to $w v$ and $w u$, which can be solved by computing the sets $M P F(w u)$ and $M P F(w v)$ (see Corollary 4.8) and checking whether they coincide or not (meaning that wu and $w v$ are or are not $\varepsilon$-twisted conjugated, respectively). Notice that, during the computations of $M P F(w u)$ and $M P F(w v)$, we can keep track of a $\varepsilon$-twisted conjugating element at each step, so that we can explicitly find a value for $x$ in the case it exists.

We remark that the full computation of the sets $M P F(w u)$ and $M P F(w v)$ will usually not be necessary. We can start the construction of both sets simultaneously, and kill the whole process giving a positive answer, as soon as we find an element $z$ in common in both sets (since, in this case, both $w u$ and $w v$ are $\varepsilon$-twisted conjugated to $z$, an so to each other).

\section{The Conjugacy Problem for some extensions of $B_{n}$.}

Theorem 5.1. Every finitely generated subgroup $A \leqslant A u t\left(B_{n}\right)$ is orbit decidable. 
Proof. Let $\varphi_{1}, \ldots, \varphi_{m} \in A u t\left(B_{n}\right)$ be given, and consider $A=\left\langle\varphi_{1}, \ldots, \varphi_{m}\right\rangle \leqslant$ Aut $\left(B_{n}\right)$. For every $i=1, \ldots, m$, compute $w_{i} \in B_{n}$ and $\epsilon_{i}=0,1$ such that $\varphi_{i}=\gamma_{w_{i}} \varepsilon_{i}^{\epsilon_{i}}$ (see the first part of the proof of Theorem 4.9).

Given two braids $u, v \in B_{n}$ we have to decide whether or not $v$ is conjugated to $\alpha(u)$ for some $\alpha \in A$. If $\epsilon_{i}=0$ for every $i$, then $A \leqslant \operatorname{Inn}\left(B_{n}\right)$ and so, the set $\{\alpha(u) \mid \alpha \in A\}$ is a certain collection of conjugates of $u$. In this case, our problem just consists on deciding whether or not $v$ is conjugated to $u$. This is doable by Theorem 1.2.

Otherwise, the set $\{\alpha(u) \mid \alpha \in A\}$ is a certain collection of conjugates of $u$ and of $\varepsilon(u)$. In this case, our problem just consists on deciding whether or not $v$ is conjugated to either $u$ or $\varepsilon(u)$. This is again doable by two applications of Theorem 1.2.

The following theorem (and the interesting particular case expressed in the corollary below) are immediate consequences of Theorems 1.1, 4.9, and 5.1.

Theorem 5.2. Let $G=B_{n} \rtimes H$ be an extension of the braid group $B_{n}$ by a finitely generated group $H$ satisfying conditions (ii) and (iii) above (for instance, take $H$ torsion-free hyperbolic). Then, G has solvable conjugacy problem.

Corollary 5.3. For any $\varphi_{1}, \ldots, \varphi_{m} \in \operatorname{Aut}\left(B_{n}\right)$, the group

$$
\left\langle B_{n}, t_{1}, \ldots, t_{m} \mid t_{i}^{-1} \sigma t_{i}=\varphi_{i}(\sigma) \quad\left(\sigma \in B_{n}\right)\right\rangle
$$

has solvable conjugacy problem.

\section{ACKNOWLEDGEMENTS}

The authors are grateful to the Centre de Recerca Matemàtica (CRM-Barcelona), since this research was initiated in the excellent research atmosphere during participation of both authors in one of the CRM workshops. The first named author acknowledges partial support from the MEC (Spain), Junta de Andalucía and FEDER, through projects MTM2007-66929, MTM2010-19355 and P09-FQM5112. The second author gratefully acknowledges partial support from the MEC (Spain) and the EFRD (EC) through project number MTM2008-01550.

\section{REFERENCES}

[1] J. S. Birman, K. H. Ko, S. J. Lee, A new approach to the word and conjugacy problems in the braid groups. Adv. Math. 139(2) (1998), 322-353.

[2] O. Bogopolski, A. Martino, E. Ventura, Orbit decidability and the conjugacy problem for some extensions of groups, Transactions of the AMS, 362(4) (2010), 2003-2036.

[3] O. Bogopolski, A. Martino, O. Maslakova, E. Ventura, Free-by-cyclic groups have solvable conjugacy problem, Bulletin of the London Mathematical Society, 38(5) (2006), 787-794. 
[4] R. Charney, Geodesic automation and growth functions for Artin groups of finite type. Math. Ann., 301(2) (1995), 307324.

[5] J.L. Dyer, E.K. Grossman, The automorphism groups of the braid groups, Amer. J. Math., 103(6) (1981), 1151-1169.

[6] E. A. El-Rifai, H. R. Morton. Algorithms for positive braids, Quart. J. Math. Oxford Ser., (2) 45(180) (1994), 479-497.

[7] N. Franco, J. González-Meneses, Conjugacy problem in braid groups and Garside groups. J. of Algebra, 266 (1) (2003), 112-132.

[8] F. A. Garside, The braid group and other groups. Quart. J. Math. Oxford Ser., (2) 20 (1969), 235-254.

[9] V. Gebhardt, A new approach to the conjugacy problem in Garside groups. J. Algebra, 292(1) (2005), 282-302.

[10] V. Gebhardt, J. González-Meneses, The cyclic sliding operation in Garside groups. Math. Z., 265(1) (2010), 85-114.

[11] J. González-Meneses, Improving an algorithm to solve multiple simultaneous conjugacy problems in braid groups. Contemp. Math., 372 (2005), 35-42.

[12] S. J. Lee and E. Lee, Potential weaknesses of the commutator key agreement protocol based on braid groups. L.R. Knudsen (Ed.): EUROCRYPT 2002, LNCS 2332, pp. 14-28, 2002.

[13] C.F. Miller III, On group-theoretic decision problems and their classification, Annals of Math., Studies 68, (1971).

[14] K. Reidemeister, Automorphismen von homotopiekettenringen, Math. Ann., 112 (1936), 586-593.

[15] Epstein et al. Word processing in groups. Jones and Bartlett Publishers, Boston, MA, 1992.

\author{
JuAn GonzÁLez-Meneses \\ DeP. Álgebra \\ UnIVERSIDAD DE SEVILLA \\ APDO. 1160 \\ 41080 Sevilla, Spain \\ E-mail address: meneses@us.es \\ ENRIC Ventura \\ Dept. Mat. Apl. III \\ Universitat Politècnica de Catalunya \\ ManResa, Barcelona \\ Catalunya \\ E-mail address: enric.ventura@upc.edu
}

